\title{
Gastric adenocarcinoma and proximal polyposis of the stomach
}

INSERM

\section{Source}

INSERM. (1999). Orphanet: an online rare disease and orphan drug data base. Gastric adenocarcinoma and proximal polyposis of the stomach. ORPHA:314022

Gastric adenocarcinoma and proximal polyposis of the stomach (GAPPS) is a rare hereditary gastric cancer characterized by proximal gastric polyposis and increased risk of early-onset, intestinal-type adenocarcinoma of the gastric body, with no duodenal or colorectal polyposis. 